\title{
AN ENTROPY-CORRECTION FREE SOLVER FOR NON-HOMOGENEOUS SHALLOW WATER EQUATIONS*
}

\author{
Tomás Chacón Rebollo ${ }^{1}$, Antonio Domínguez Delgado ${ }^{2}$ \\ AND EnRique D. FERnÁNDEZ Nieto ${ }^{3}$
}

\begin{abstract}
In this work we introduce an accurate solver for the Shallow Water Equations with source terms. This scheme does not need any kind of entropy correction to avoid instabilities near critical points. The scheme also solves the non-homogeneous case, in such a way that all equilibria are computed at least with second order accuracy. We perform several tests for relevant flows showing the performance of our scheme.
\end{abstract}

Mathematics Subject Classification. 65N06, 76B15, 76M20, 76N99.

Received: November 18, 2002. Revised: March 27, 2003.

\section{INTRODUCTION}

In this work we consider numerical approximations to 1D Shallow Water Equations (SWE), with source terms. These equations are a system of nonlinear hyperbolic partial differential equations that can be written in generalized conservation form as

$$
\begin{aligned}
& \left.\frac{\partial}{\partial t} W+\frac{\partial}{\partial x} F(W)=G(x, W) \quad \text { in }\right] 0, L[\times] 0, T[ \\
& \text { Here the unknown is } W(x, t)=\left(\begin{array}{c}
h(x, t) \\
q(x, t)
\end{array}\right) ;
\end{aligned}
$$

where $h(x, t)$ and $q(x, t)$ respectively denote the height of the water column and the discharge across the channel

Keywords and phrases. Finite Volume Method, upwinding, shallow water, Harten Regularization, source terms, entropycorrection

* This research was partially supported by Spanish Government Research Projects REN2000-1162-C02-01 and REN20001168-C02-01.

${ }^{1}$ Departamento de Ecuaciones Diferenciales y Análisis Numérico, Universidad de Sevilla, C/ Tarfia, s/n, 41080 Sevilla, Spain. e-mail: chacon@numer.us.es

2 Departamento de Matemática Aplicada I, Universidad de Sevilla, E.T.S. Arquitectura Avda, Reina Mercedes s/n, 41012 Sevilla, Spain. e-mail: domdel@us.es

${ }^{3}$ Departamento de Matemática Aplicada I, Universidad de Sevilla, E.T.S. Arquitectura. Avda, Reina Mercedes, s/n, 41012 Sevilla, Spain. e-mail: edofer@us.es 
section at position $x \in] 0, L[$ and time $t \in] 0, T[. F$ denotes the physical flux, given by:

$$
F(W)=\left(\begin{array}{c}
q \\
\frac{q^{2}}{h}+\frac{1}{2} g h^{2}
\end{array}\right),
$$

where $g$ denotes the gravity constant. $G$ is the source term, which may take into account variable depth and width of the channel, and also friction effects. The precise definition of $G$ shall be given later on.

We shall consider finite volume numerical solvers. Given a cell size $\Delta x$ for the spatial discretization and a time step $\Delta t$ for the time discretization, we define $x_{j}=j \Delta x$ and $t^{m}=m \Delta t$. Then, we shall approximate the solution $W(x, t)$ of (1) in $t=t^{n}$ by a piecewise constant function that takes the constant value $W_{i}^{n}$ on the cell ]$x_{i-1 / 2}, x_{i+1 / 2}\left[\right.$. The values $\left\{W_{i}^{n}\right\}_{n, i}$ for the homogeneous system are obtained as the solution of general explicit solvers of the form

$$
\frac{W_{i}^{n+1}-W_{i}^{n}}{\Delta t}=-\frac{\phi\left(W_{i}^{n}, W_{i+1}^{n}\right)-\phi\left(W_{i-1}^{n}, W_{i}^{n}\right)}{\Delta x}
$$

complemented with suitable initial and boundary conditions. The scheme is defined through some specific choice of the numerical flux function $\phi\left(W_{i}, W_{i+1}\right)$.

In this paper we address the problem set by the lack of numerical viscosity for upwind schemes due to the presence of critical points where the Froude number, equal to $\mathcal{F}=|q / h| / \sqrt{g h}$, takes on specific values. At these points, one of the eigenvalues of the Jacobian matrix of the flux $F$ vanishes, thus producing a lack of numerical viscosity. This generates a loss of accuracy that propagates up and downstream, and may give completely non-physical (non-entropy) solutions ( $c f .[1,9,15,22])$. In the case of Shallow Water Equations for flux-difference schemes, such critical points are the sonic ones, corresponding to $\mathcal{F}=1$, while for flux-splitting schemes, the critical points correspond to $\mathcal{F}=1 / \sqrt{2}$.

To avoid this situation, several entropy corrections have been proposed to add some artificial viscosity near critical points. One of the commonly used technique of regularization is due to Harten (cf. [11]). This is a very extended technique that stabilizes the computation near critical points for general hyperbolic conservations systems. However, this technique involves the use of some parameters whose values must be tuned in an empirical way.

There are some other techniques (cf. [10]) that do not contain any arbitrary parameter, but in general, they are of more complicated use and the Harten's regularization is, in spite of all, the most used way to introduce entropy corrections in the upwind schemes.

In this paper we introduce a stable scheme for SWE with source terms, which does not require any kind of entropy correction.

The basic idea to build our scheme is to upwind the numerical flux function in such a way that the eigenvalues of the upwinding matrix become zero only in the supercritical area. Therefore, when an instability, caused by the vanishing of some eigenvalue of the upwinding matrix, appears, it cannot propagate upstream.

This procedure leads to a numerical scheme simple to implement, that behaves as Roe's or Van Leer's methods with optimal Harten's regularization. Moreover it requires a small computational cost, similar to that of Roe's without Harten's regularization.

We also derive an equilibria - preserving extension of our scheme to non-homogeneous SWE. It is known that to be accurate enough for the non-homogeneous equations, the scheme should solve some suitable equilibrium solution at least up to second order. Otherwise, physically unacceptable solutions are computed due to very large errors (cf. $[20,21])$.

There is a considerable litterature addressing this question. In [18] Roe presents some contributions to the study of non-homogeneous hyperbolic conservations laws. He relates the election of quadratura formulae for the source terms with the property that the scheme calculates up to second order the stationnary state.

Bermúdez and Vázquez, in [20,21] formalised this property by asking that the numerical solution exactly (or approximately up to second order) solves some steady solutions at grid nodes ("C-property of conservation"). Extensions of Roe's and Van-Leer's schemes satisfying this property are derived in [20,21]. 
Le Veque, in [16] introduced a quasi-steady wave propagation algoritm. This is based upon the solution of Riemann problems with modified constant states, in such a way that the flux difference exactly balances the source term.

Jin, in [12] introduce the interface method, which preserve the stationary state solutions for SWE at cellinterfaces. In this work, some stationary solutions analysed are not at rest.

Kurganov and Levy, in [13], introduce an extension of the central-upwind scheme presented by Kurganov and Tadmor in [14]. These schemes are characterised by requiring only a scalar viscosity to ensure stability, instead of a matrix viscosity as is usual for flux difference and flux splitting schemes. Its extension to non-homogeneous SWE solves a particular stationary solution for a re-formulation of SWE where the unknowns are $(\omega, q)$, with $\omega=h+z(x)$, instead of the conservative variables $(h, q)$, where $h$ is the height of the flow, $q$ is the discharge and $z(x)$ gives the bottom profile.

Perthame and Simeoni, in [17], use a kinetic approach to SWE. Under reasonable CFL conditions they prove non-negativity of the height of water and an entropy inequality for the numerical fluxes. Moreover some steady states are proved to be calculated exactly.

In [6] an extension of a flux-splitting scheme to non-homogeneous $1 \mathrm{D}$ SWE is derived. In [7] a systematic technique, based upon the study introduced in [6], is defined to build solvers which verify the C-property defined by Bermúdez and Vázquez. The idea in [7] is to separately compensate the centered and decentered components of the numerical flux by specific discretizations of the source term. This scheme is proved to exactly solve some relevant known equilibria.

In this paper we focus the study of the extension to the non-homogeneous case of numerical schemes that can be formulated as a numerical viscosity method (see [9]).

Our analysis is based upon the construction of an adequate equivalent PDE system. We recall that the equivalent system is a modification of the SWE (in our case) by adding the diffusion terms associated to the scheme and for which the numerical scheme is a second order approximation.

The key point is to build up an equivalent system such that all equilibria of SWE also are equilibria of the equivalent system. Then, any second order approximation of this system solves up to second order the equilibria of the original SWE. So, if we define the numerical scheme as a second order approximation in space of this equivalent system, we ensure that it calculates up to second order all equilibria of the original system. Moreover we do not need to explicitely know the expression of equilibria.

We consider extensions of general flux splitting and flux difference schemes, but our analysis can be extended to any scheme which can be re-formulated as an artificial viscosity method. This is the case, for instance, of the scheme introduced in [13].

The paper is organised as follows: in Section 2 we present the numerical scheme that is introduced for the homogeneous SWE. Some numerical tests are carried on by comparison with either analytical solutions or highly performing already known methods. In Section 3 we build the equilibria - preserving equivalent system for general non-homogeneous conservation laws. In Section 4 we present the extension of our actual method to the non homogeneous SWE as a particular case of the derivation set in Section 3. Also we perform several tests for relevant flows. We test energy conservation for steady solutions not at rest, stable computations of dry zones and transcritical flows, all of them produced by bottom gradients. In particular, in all transcritical cases we obtain results quite close to Roe's or Van Leer's schemes with optimal Harten regularization, obtaining gains for some specific flows (see Test 7).

\section{Homogeneous equations}

To describe the scheme that we introduce, we start from a general upwind numerical scheme, with the structure

$$
\phi\left(W_{i}, W_{i+1}\right)=\frac{F\left(W_{i}\right)+F\left(W_{i+1}\right)}{2}-\frac{1}{2}\left(\left|D_{i+1}\right| W_{i+1}-\left|D_{i}\right| W_{i}\right),
$$

where $D$ is the upwinding matrix and $|D|$ represents its "absolute value" (cf. [19]). 
Both flux-difference and flux-splitting schemes fit into the general structure of (5). Flux-difference schemes use the Jacobian matrix $A$ of the flux $F$ to build the upwinding matrix $D(c f$. [5]), while flux-splitting schemes use the matrix $\mathcal{A}^{*}$ that satisfies $F(W)=\mathcal{A}^{*}(W) W(c f$. [6]); given by

$$
\mathcal{A}^{*}(U)=\left(\begin{array}{cc}
0 & 1 \\
-\frac{q^{2}}{h^{2}}+\frac{1}{2} g h & 2 \frac{q}{h}
\end{array}\right) .
$$

For instance, Van Leer's scheme is defined by

$$
D_{i+1}=D_{i}=A\left(W_{i+1 / 2}\right) \quad \text { with } \quad W_{i+1 / 2}=\frac{W_{i}+W_{i+1}}{2},
$$

while Steger-Warming's scheme corresponds to

$$
D_{i+1}=\mathcal{A}^{*}\left(W_{i+1}\right), \quad D_{i}=\mathcal{A}^{*}\left(W_{i}\right) .
$$

We propose to use as upwinding matrix $D_{i}=D_{i+1}=\widehat{A}\left(W_{i+1 / 2}\right)$, with $\widehat{A}$ defined by:

$$
\widehat{A}=\sigma A+(1-\sigma) \mathcal{A}^{*},
$$

where $\sigma$ is in principle a free parameter.

This can be interpreted as a hybrid flux-difference - flux-splitting scheme.

For a convenient choice of $\sigma$, the eigenvalues of $\widehat{A}$ vanish only in the supercritical area. Indeed, the eigenvalues of $\widehat{A}$ are

$$
\widehat{\lambda}_{1}=\frac{q}{h}+(1+\delta) \sqrt{g h}, \quad \widehat{\lambda}_{2}=\frac{q}{h}-(1+\delta) \sqrt{g h},
$$

where $\delta$ is obtained from $\sigma$ by the relation $\sigma=2(1+\delta)^{2}-1$. The associated eigenvectors are

$$
e_{1}=\left(\begin{array}{c}
1 \\
\widehat{\lambda}_{1}
\end{array}\right), \quad e_{2}=\left(\begin{array}{c}
1 \\
\widehat{\lambda}_{2}
\end{array}\right)
$$

For $\delta>0$, if $q \geq 0$ then $\widehat{\lambda}_{1}$ is always positive and $\widehat{\lambda}_{2}=0$ only if $\mathcal{F}=1+\delta$, corresponding to supercritical flow.

The actual numerical scheme that we propose is given by the flux function

$$
\phi\left(W_{i}, W_{i+1}\right)=\frac{F\left(W_{i+\alpha}\right)+F\left(W_{i+1-\alpha}\right)}{2}-\frac{1}{2}\left|\widehat{A}\left(W_{i+1 / 2}\right)\right|\left(W_{i+1}-W_{i}\right),
$$

where the evaluation point for the flux is given by

$$
W_{i+\alpha}=(1-\alpha) W_{i}+\alpha W_{i+1} \quad \text { with } \quad 0 \leq \alpha \leq \frac{1}{2} .
$$

The scheme that we propose corresponds to $\delta=0.05$. This choice allows to reach the supercritical area, to avoid the propagation of spurious oscillations, but without introducing an excessive diffusion that could damage the accuracy of the scheme. Also, for reasons of equilibrium between stability and accuracy we shall take $\alpha=1 / 8$. We shall keep the same values of $\alpha$ and $\delta$ for all the numerical tests that we present.

Remark 1. Choosing the centered part of the numerical flux as

$$
\frac{F\left(W_{i+\alpha}\right)+F\left(W_{i+1-\alpha}\right)}{2}
$$




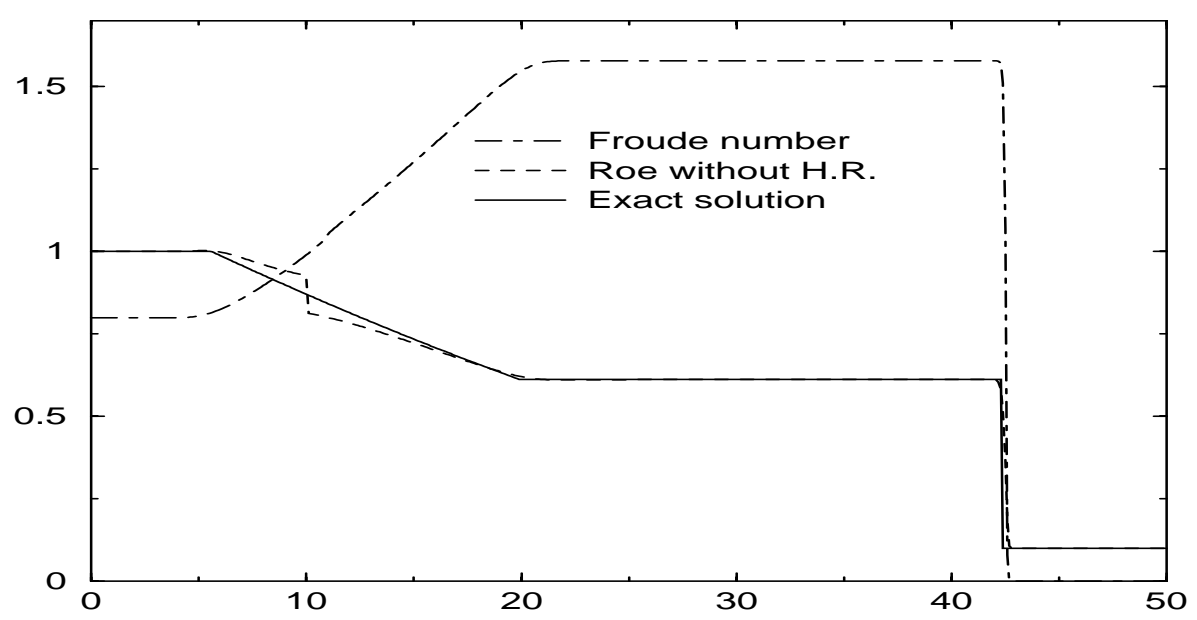

FiguRE 1. Test 1: Roe-Van Leer without Harten regularization.

reduces the virtual cone of dependence of the scheme. The largest of such virtual cones is $\left[x_{i-1}, x_{i+1}\right]$ corresponding to $\alpha=0$; and the smallest is $\left[x_{i-1 / 2}, x_{i+1 / 2}\right]$, corresponding to $\alpha=1 / 2$. In the first case we obtain a more diffusive (but stable) scheme while in the second the numerical difussion is reduced to a minimum, but the schemes is less stable. Then, when $\alpha$ is near $1 / 2$ some problems of stability can appear in shocks, whereas smooth flow regions are well approximated. Our scheme can also be viewed as a Godunov type scheme for which the constant values associated to the Riemann problem in $x_{i+1 / 2}$ are approximations of $W$ in $x_{i+\alpha}$ and $x_{i+1-\alpha}$ ( $W_{i+\alpha}$ and $W_{i+1-\alpha}$ respectively).

The choice $\alpha=1 / 8$ in practice allows to reduce the levels of numerical diffusion, while keepping the stability of the scheme. This choice has been deduced from several numerical tests with $\delta$ of order $10^{-2}$ and $\alpha \in[1 / 8,3 / 8]$ by comparing the behaviour of the scheme in shocks and rarefactions waves.

Remark 2. Scheme (7) can be directly extended to finite volume solvers of 2D SWE. This scheme has been tested with success for genuinely $2 \mathrm{D}$ flows. This extension is currently being written as a part of a scientific paper.

We next present two tests for homogeneous SWE. These are two Riemann problems where the difficulties for the numerical solvers are due to the presence of a rarefaction wave and of a shock wave.

The initial conditions are set to produce rarefaction waves containing critical points with Froude numbers $\mathcal{F}=1$ for Test 1 and $\mathcal{F}=1 / \sqrt{2}$ for Test 2 . We compare our solver with a flux diference solver in Test 1 and a flux splitting solver for Test 2. In both tests the Froude number reaches values strictly larger that 1 , theoretically critical for our scheme.

In both Tests we have considered a domain length $L=50$ meters, $\Delta x=1$ and a C.F.L condition equal to 0.8 . The discontinuity of the initial condition is located at $x=10$.

Test 1. The Froude number crosses the value one

Here, we have considered the following Riemann problem as initial condition:

$$
W(x, 0)=\left\{\begin{array}{lll}
h=1 \mathrm{~m}, & q=2.5 \mathrm{~m}^{2} / \mathrm{s} & \text { if } x<10 \\
h=0, & q=0 & \text { if } x>10
\end{array}\right.
$$

We present our results for $t=7 \mathrm{~s}$.

In Figure 1 we compare the computed free surface from Roe's scheme versus the exact solution. In this case Van Leer's scheme provides a solution quite close to Roe's scheme. 


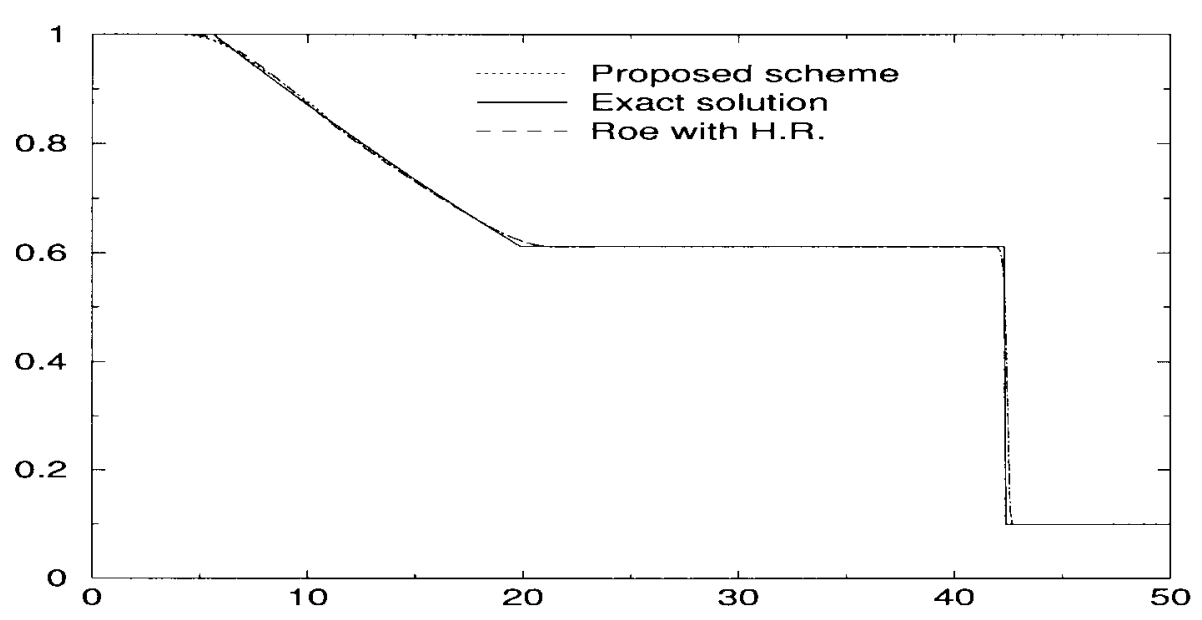

Figure 2. Test 1: Proposed scheme versus exact solution and Roe's scheme with Harten regularization.

We can observe the "dog leg" profile (false shock) that appears when the Froude number crosses the value one and that generates errors upstream and downstream.

In Figure 2 we represent the results that Roe's scheme provide applying Harten regularization. We recall that this regularization consist in substituting $\left|\lambda_{i}\right|$ by

$$
\left|\lambda_{i}\right|_{\varepsilon}=\left|\lambda_{i}\right|+\frac{1}{2}\left(1+\operatorname{sgn}\left(\varepsilon-\left|\lambda_{i}\right|\right)\right)\left(\frac{\lambda_{i}^{2}+\varepsilon^{2}}{2 \varepsilon}-\left|\lambda_{i}\right|\right), \quad i=1,2
$$

where $\varepsilon=\epsilon \sqrt{g h}$, with $\epsilon$ a small positive parameter whose actual value should be tuned for each particular flow (cf. [4]). A simpler possibility is to replace $\lambda_{i}$ by

$$
\operatorname{sgn}\left(\lambda_{i}\right) \max \left(\left|\lambda_{i}\right|, \epsilon\right)
$$

however, this produces excesive numerical diffusion and its tuning is largely dependent of the actual flow.

In [2] the regularization of Harten is applied to the scheme of Roe by taking $\epsilon=0.5$. This is the value that we use to compute the solution presented in Figure 2.

We remark that no instabilities or loss of precision appear in the result of the proposed scheme, despite the fact that at some point in the rarefaction wave the Froude number takes the value $\mathcal{F}=1.05$, where one of the eigenvalues of matrix $\widehat{A}$ vanishes.

Test 2. The Froude number crosses the value $1 / \sqrt{2}$

This second test is defined with the same geometrical data as the previous one. As initial condition we consider the Riemann problem

$$
W(x, 0)=\left\{\begin{array}{lll}
h=1, & q=0 & \text { if } x<10 \\
h=0, & q=0 & \text { if } x>10
\end{array}\right.
$$

We present our results for $t=4 \mathrm{~s}$. In Figure 3 we represent the result of the flux-splitting defined by (5) with $D_{i}=D_{i+1}=\mathcal{A}^{*}\left(W_{i+/ 2}\right)$. An instability spreads downstream from the point where an eigenvalue of the upwinding matrix corresponding to this scheme becomes zero, that is, from the value $\mathcal{F}=1 / \sqrt{2}$. Moreover we observe some oscillations near the shock. For a smaller CFL number, the oscillations dissapear, but the inaccuracy that spreads from the point where $\mathcal{F}=1 / \sqrt{2}$ increases (see Fig. 4). 


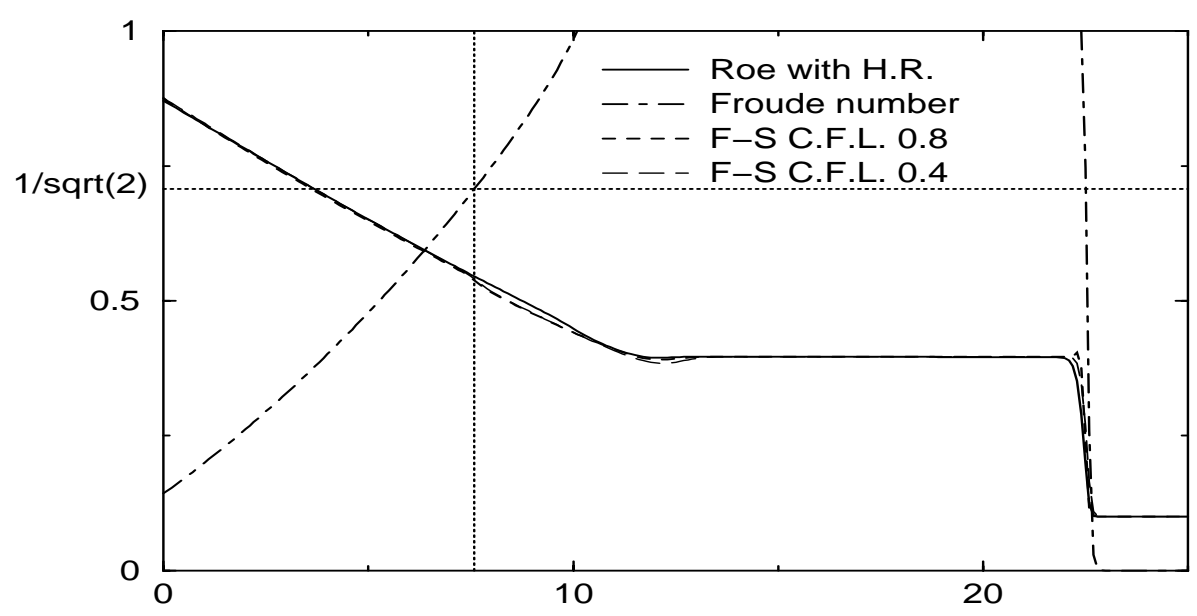

Figure 3. Test 2: Flux-splitting method.
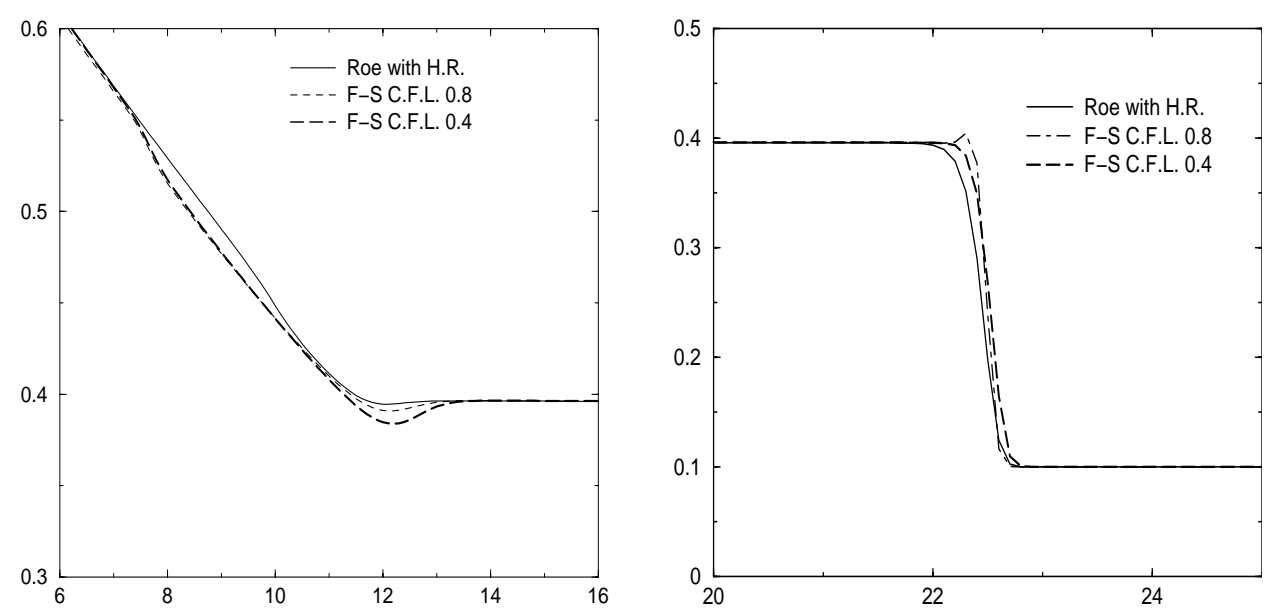

FiguRE 4. Test 2: Flux-splitting method: rarefaction wave and shock wave.

In Figure 5 we compare the exact solution versus the proposed scheme. The results are still very close for this second test, as for Test 1.

Therefore, we can conclude that for homogeneous SWE, the proposed scheme yields good performances in the cases where flux-difference and flux-splitting schemes fail due to the vanishing of the eigenvalues of their respective upwinding matrix; providing results quite close to the use of optimized Harten's regularization.

\section{TREATMENT OF SOURCE TERMS FOR GENERAL HYPERBOLIC SYSTEMS}

Efficient solvers of non-homogeneous systems should provide high-precision approximation of equilibria solutions. Otherwise, large errors can appear in the computed solution ( $c f$. [1]). In this section we give simple sufficient conditions that imply this "enhanced consistency" property for all equilibria solutions, without the need of calculating any of them. This will motivate our discretization for the source terms. 


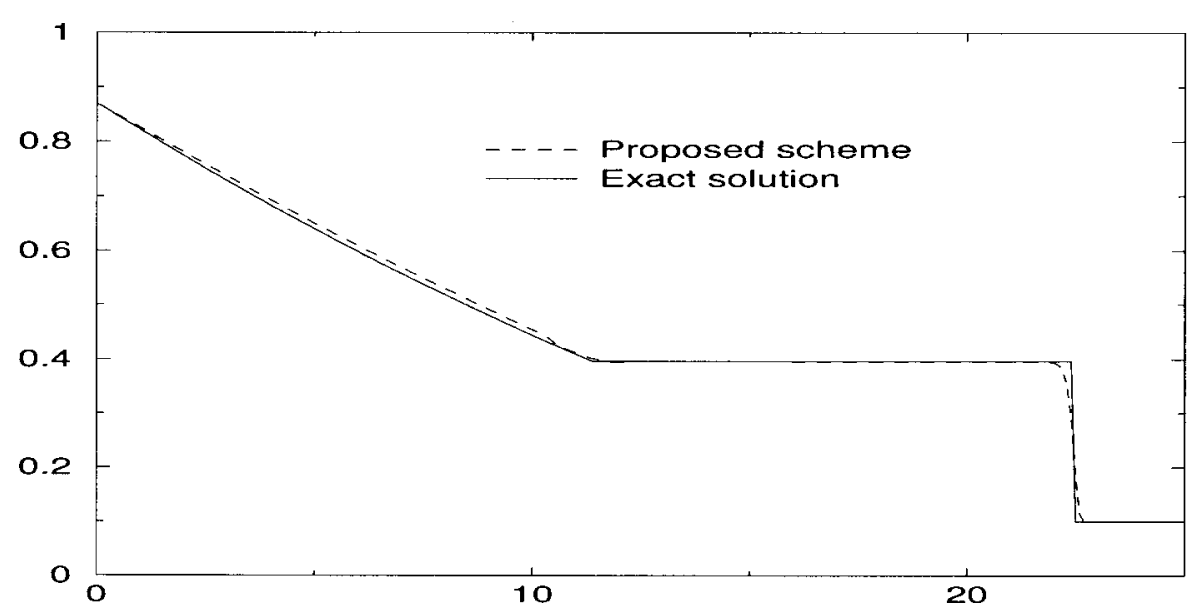

Figure 5. Test 2: Proposed scheme versus exact solution.

We observe that in the case of homogeneous hyperbolic systems, as

$$
\frac{\partial W}{\partial t}+\frac{\partial}{\partial x} F(W)=0
$$

the numerical schemes that use upwinding matrix, can be interpreted as centered discretizations, (cf. [10] and $[15]$ ), of the equivalent convection-diffusion equation,

$$
\frac{\partial W}{\partial t}+\frac{\partial}{\partial x} F(W)-\nu \frac{\partial}{\partial x}\left(|D(W)| \frac{\partial}{\partial x} W\right)=0
$$

where $\nu$ is equal to half of the space step $(\nu=\Delta x / 2)$ and $|D|$ is the numerical diffusion matrix. Flux splitting and flux difference schemes fit into this framework. In particular our actual scheme, for which $D=\widehat{A}$.

Notice that the constant states $W$, which are all the smooth steady solutions of the hyperbolic system (8), also are steady solutions of the equivalent system (9). Thus, for the homogeneous system the enhanced consistency conditions is automatically satisfied.

Next, we consider a hyperbolic system with source terms, as:

$$
\frac{\partial W}{\partial t}+\frac{\partial}{\partial x} F(W)=G(x, W)
$$

If we apply a centered discretization to the source term, then the equivalent system is

$$
\frac{\partial W}{\partial t}+\frac{\partial}{\partial x} F(W)-\nu \frac{\partial}{\partial x}\left(|D(W)| \frac{\partial}{\partial x} W\right)=G(x, W) .
$$

Thus, we are approximating steady solutions with a first order error.

Instead, we propose a discretization of the source term that contains a "viscous" correction of the source term,

$$
\frac{\partial W}{\partial t}+\frac{\partial}{\partial x} F(W)-\nu \frac{\partial}{\partial x}\left(|D(W)| \frac{\partial}{\partial x} W\right)=G(x, W)+\text { VST. }
$$

Our goal is to tune the viscosity source term, VST, so that all equilibria solutions of the hyperbolic system (10) also are solutions of the equivalent equation (11). Any centered approximation (in space) of (11) will solve up to second order all equilibria solutions of (11) and thus all equilibria solutions of (10). Also, higher order approximations of (11) will solve the equilibria of (10) with the same accuracy order. 
To construct our correction term, let us consider a smooth equilibrium solution $W$ of (10), then it verifies:

$$
A(W) \frac{\partial W}{\partial x}=\frac{\partial}{\partial x} F(W)=G(x, W)
$$

Therefore, if $A(W)$ is not singular,

$$
\frac{\partial W}{\partial x}=A^{-1}(W) G(x, W)
$$

and then,

$$
\frac{\partial}{\partial x}\left(|D(W)| \frac{\partial}{\partial x} W\right)=\frac{\partial}{\partial x}\left[|D(W)| A^{-1}(W) G(x, W)\right] .
$$

According to (11), the correction term should balance $-\nu \frac{\partial}{\partial x}\left(|D| \frac{\partial}{\partial x} W\right)$, and in consequence it must be given by

$$
\operatorname{VST}=-\nu \frac{\partial}{\partial x}\left(|D| A^{-1} G\right)
$$

Thus, (11) is re-written as

$$
\frac{\partial W}{\partial t}+\frac{\partial}{\partial x} F(W)+\frac{\partial}{\partial x}\left(-\nu|D(W)| \frac{\partial}{\partial x} W\right)=G(x, W)-\nu \frac{\partial}{\partial x}\left[|D(W)| A^{-1}(W) G(x, W)\right] .
$$

Now, it is enough to construct centered approximations of each term in (13) (excepting the time derivative) to achieve a method that approximates the equilibria of system (13) up to second order.

This technique can be applied to any scheme that can be written in viscous form. As a particular case we apply it to the scheme defined for the homogeneous system in the previous section.

Remark 3. To analyse the case where $A$ is not inversible we consider a smooth stationnary solution $W(x)$ which has a sonic point $x_{0}$. If this point is isolated (there are no other sonic points in a neighbourhood of $x_{0}$ ) then $A(W(x))$ is inversible in this neighbourhood, excepting $x_{0}$. Then, we have in these points

$$
\frac{\partial}{\partial x}\left(|D(W(x))| A^{-1}(W(x)) G(x, W(x))\right)=\frac{\partial}{\partial x}\left(|D(W(x))| \frac{\partial W(x)}{\partial x}\right) .
$$

Consequently the correction term has a limit in $x_{0}$ and, in fact, is continuous in $x_{0}$.

Remark 4. Notice that system (13) can be interpreted as a viscous approximation to the modified conservation system

$$
\frac{\partial W}{\partial t}+\frac{\partial}{\partial x}(F(W)+S(x, W))=G(x, W)
$$

with $S(x, W)=\nu D(W) A^{-1}(W) G(x, W)$.

That is, the new term $V S T$ can ve viewed as a numerical modification to the flux. It does not introduce any modification in the diffusion term,

$$
-\nu \frac{\partial}{\partial x}\left(|D(W)| \frac{\partial}{\partial x} W\right)
$$

One may wonder whether for this modified flux the numerical diffusion, when $D(W)=\widehat{A}(W)$, still vanishes in supercritical flow zones. In fact, this occurs in supercritical zones associated to the flux $F(W)$ because the difussion matrix $D(W)$ is constructed with the eigenvalues associated to this flux. It would not have a physical meaning to use the modified flux $F(W)+S(W)$ to construct the diffusion matrix $D(W)$. 
Remark 5. The technique that we present of equilibria preserving extensions can be applied to general schemes that may be re-formulated as artificial viscosity methods. For instance, this is the case of the scheme proposed in [14]. In this case, the equivalent equation is

$$
\frac{\partial W}{\partial t}+\frac{\partial}{\partial x} F(W)+\frac{\partial}{\partial x}\left(\nu_{1}(W) \frac{\partial W}{\partial x}\right)+\frac{\partial}{\partial x}\left(\nu_{2}(W) \frac{\partial F(W)}{\partial x}\right)=G(x, W)
$$

for some appropiate $\nu_{1}(W)$ and $\nu_{2}(W)$.

\section{Application to shallow Water equations}

In this section we consider the SWE with source term $G$ due to variable depth:

$$
G(x,(h, q))=z^{\prime}(x)\left(\begin{array}{l}
g_{1} \\
g_{2}
\end{array}\right)(W)
$$

where $z(x)$ describes the bottom of the channel and $g_{1}(W)=0, g_{2}(W)=-g h$. The treatment of the source terms taking into account variable width of the channel and friction effects, is carried on in a similar way, but for brevity we omit them.

For SWE, the proposed scheme (7) for the homogeneous case, discretizes the diffusion flux term

$$
-\nu \frac{\partial}{\partial x}\left(|\widehat{A}(W)| \frac{\partial}{\partial x} W\right)
$$

at $x=x_{i}$ by

$$
-\nu \frac{\left|\widehat{A}\left(W_{i+1 / 2}\right)\right|\left(W_{i+1}-W_{i}\right) / \Delta x-\left|\widehat{A}\left(W_{i-1 / 2}\right)\right|\left(W_{i}-W_{i-1}\right) / \Delta x}{\Delta x} .
$$

Then, we propose a discretization with a similar structure to (16) for the diffusion term that appears in the right hand side of (13):

$$
\begin{aligned}
-\left.\nu \frac{\partial}{\partial x}\left[|\widehat{A}(W)| A^{-1}(W) G(x, W)\right]\right|_{x=x_{i}} & \simeq \\
& \frac{-\nu}{\Delta x}\left[\left|\widehat{A}\left(W_{i+1 / 2}\right)\right| A^{-1}\left(W_{i+1 / 2}\right) G_{i+1 / 2}-\left|\widehat{A}\left(W_{i-1 / 2}\right)\right| A^{-1}\left(W_{i-1 / 2}\right) G_{i-1 / 2}\right]
\end{aligned}
$$

where $G_{i+1 / 2}$ and $G_{i-1 / 2}$, should be approximations of first order of $G\left(x_{i+1 / 2}, W\left(x_{i+1 / 2}\right)\right)$ and $G\left(x_{i-1 / 2}\right.$, $\left.W\left(x_{i-1 / 2}\right)\right)$, respectively.

Concretely, we propose the following scheme,

$$
\begin{aligned}
& W_{i}^{n+1}=W_{i}^{n}-\frac{\Delta t}{\Delta x}(\left.\phi\left(W_{i}^{n}, W_{i+1}^{n}\right)-\phi\left(W_{i-1}^{n}, W_{i}^{n}\right)\right) \\
& \quad+\frac{\Delta t}{2}\left(G_{L}\left(W_{i-1}^{n}, W_{i}^{n}\right)+G_{R}\left(W_{i}^{n}, W_{i+1}^{n}\right)\right)-\frac{\Delta t}{\Delta x}\left(V\left(W_{i}^{n}, W_{i+1}^{n}\right)-V\left(W_{i-1}^{n}, W_{i}^{n}\right)\right)
\end{aligned}
$$

where the different elements are defined as follows. 
Firstly we observe that to define $V\left(W_{i}, W_{i+1}\right)$, we use the inverse matrix of $A$. Using Remark 3 , we propose to replace $A^{-1}$ by a correction $\overline{A^{-1}}$ differently defined only in a neighbourhood of sonic points:

$$
\overline{A^{-1}}=X \overline{\Lambda^{-1}} X^{-1}, \quad \text { where } \overline{\Lambda^{-1}}=\left(\begin{array}{cc}
\overline{\lambda_{1}^{-1}} & 0 \\
0 & \overline{\lambda_{2}^{-1}}
\end{array}\right), \quad \text { with } \quad \overline{\lambda_{i}^{-1}}=\left\{\begin{array}{ccc}
1 / \lambda_{i} & \text { if } & \left|\lambda_{i}\right|>\epsilon \\
0 & \text { if } & \left|\lambda_{i}\right| \leq \epsilon
\end{array} \quad i=1,2,\right.
$$

where $\epsilon$ is close to the computer precision.

Then we define the different components of (18) as follows:

a) To define $V\left(W_{i}, W_{i+1}\right)$, we use matrix $\overline{A^{-1}}$,

$$
V\left(W_{i}, W_{i+1}\right)=\frac{\Delta x}{2}\left|\widehat{A}\left(W_{i+1 / 2}\right)\right| \overline{A^{-1}}\left(W_{i+1 / 2}\right) G_{i+1 / 2}
$$

with

$$
G_{i+1 / 2}=\frac{z_{i+1}-z_{i}}{\Delta x}\left(\begin{array}{c}
g_{1} \\
g_{2}
\end{array}\right)\left(W_{i+1 / 2}\right)
$$

b) $G_{L}\left(W_{i-1}, W_{i}\right)$ and $G_{R}\left(W_{i}, W_{i+1}\right)$ are approximations of $G\left(x_{i}, W_{i}\right)$ in terms of the parameter $\alpha$ that determines the centered component of the numerical flux (7). Their actual definition is:

$$
\begin{aligned}
G_{L}\left(W_{i-1}, W_{i}\right)= & \frac{x_{i}-x_{i-1+\alpha}}{\Delta x}\left(\begin{array}{c}
g_{1} \\
g_{2}
\end{array}\right)\left(\frac{W_{i}+W_{i-1+\alpha}}{2}\right) \frac{z_{i}-z_{i-1+\alpha}}{x_{i}-x_{i-1+\alpha}} \\
& +\frac{x_{i}-x_{i-\alpha}}{\Delta x}\left(\begin{array}{c}
g_{1} \\
g_{2}
\end{array}\right)\left(\frac{W_{i}+W_{i-\alpha}}{2}\right) \frac{z_{i}-z_{i-\alpha}}{x_{i}-x_{i-\alpha}}, \\
G_{R}\left(W_{i}, W_{i+1}\right)= & \frac{x_{i+\alpha}-x_{i}}{\Delta x}\left(\begin{array}{c}
g_{1} \\
g_{2}
\end{array}\right)\left(\frac{W_{i}+W_{i+\alpha}}{2}\right) \frac{z_{i+\alpha}-z_{i}}{x_{i+\alpha}-x_{i}}+ \\
& +\frac{x_{i+1-\alpha}-x_{i}}{\Delta x}\left(\begin{array}{c}
g_{1} \\
g_{2}
\end{array}\right)\left(\frac{W_{i}+W_{i+1-\alpha}}{2}\right) \frac{z_{i+1-\alpha}-z_{i}}{x_{i+1-\alpha}-x_{i}} .
\end{aligned}
$$

In principle, setting $G_{L}=G_{R}=G\left(x_{i}, W_{i}\right)$ is enough to guarantee that the scheme preserves steady-states up to second order accuray, following the analysis of the preceding section. However, our choice ensures moreover that the scheme exactly solves the equilibrium solution given by

$$
W=\left(\begin{array}{c}
\bar{h}-z(x) \\
0
\end{array}\right)
$$

where $\bar{h}$ is a constant reference height. For the sake of brevity we do not include the proof.

\subsection{Numerical tests}

In this section we present several numerical tests in domains with no plane bottom. Firstly we perform two tests for stationary solutions not at rest and compare with the cell interface method developed in [12]. Secondly we compute a test proposed in [8] where the performance of the scheme when a dry zone occurs is studied. Finally, we present two tests for transcritical flows, generated by a bottom bump.

Test 4. Stationary solutions

We perform two numerical tests proposed in [12]. They are based in the fact that

$$
\begin{gathered}
q=\text { constant } \\
\frac{1}{2}\left(\frac{q}{h}\right)^{2}+g(h+z(x))=\text { constant }
\end{gathered}
$$




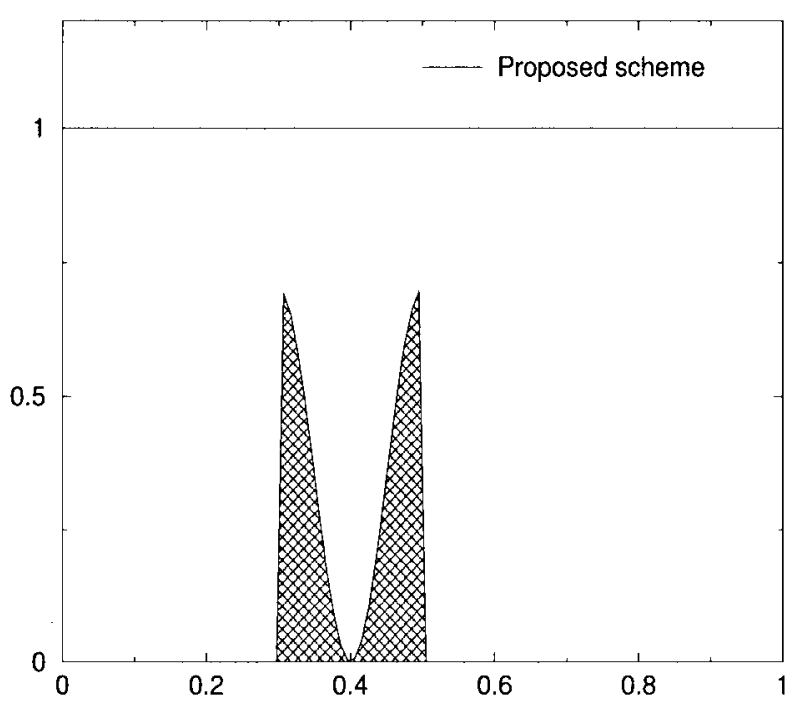

Figure 6. Test 4.1: $\frac{1}{2}\left(\frac{q}{h}\right)^{2}+g(h+z(x))$ and bottom profile.

are stationary solutions for SWE with variable depth.

For the two following sub-tests we take $g=1$ and compare the numerical solution provided by the proposed scheme with (20).

The spatial domain is $[0,1]$, we take $\Delta x=0.01$ and a C.F.L. condition equal to 0.8 .

Sub-test 4.1. Stationary solution at rest

In this case, the bottom function is

$$
z(x)=\left\{\begin{array}{cc}
0.35 \times(\cos (\pi(x-0.5) / 0.1)+1) & 0.3<x<0.5 \\
0 & \text { otherwise }
\end{array}\right.
$$

We consider as boundary condition $\partial_{x} q=0$ and $\partial_{x} h=0$ at $x=0$ and $x=1$.

In Figure 6 we present the profile bottom function and the numerical result that we obtain, starting from the initial condition

$$
\begin{gathered}
h_{i}=1-\frac{1}{2}\left(z_{i-1 / 2}+z_{i+1 / 2}\right) \\
q_{i}=0 .
\end{gathered}
$$

For this initial condition, we have

$$
\frac{1}{2}\left(\frac{q}{h}\right)^{2}+g(h+z(x))=1
$$

at any point excepting near the obstacle. We observe that the steady solution reached closely satisfies (21). This solution is solved up to an error of $10^{-5}$ in $L^{\infty}$ norm.

Sub-test 4.2. Stationary solution not at rest

In this case, the bottom function is

$$
z(x)=\left\{\begin{array}{cc}
0.35 \times(\cos (\pi(x-0.5) / 0.1)+1) & 0.4<x<0.5 \\
0 & \text { otherwise }
\end{array}\right.
$$




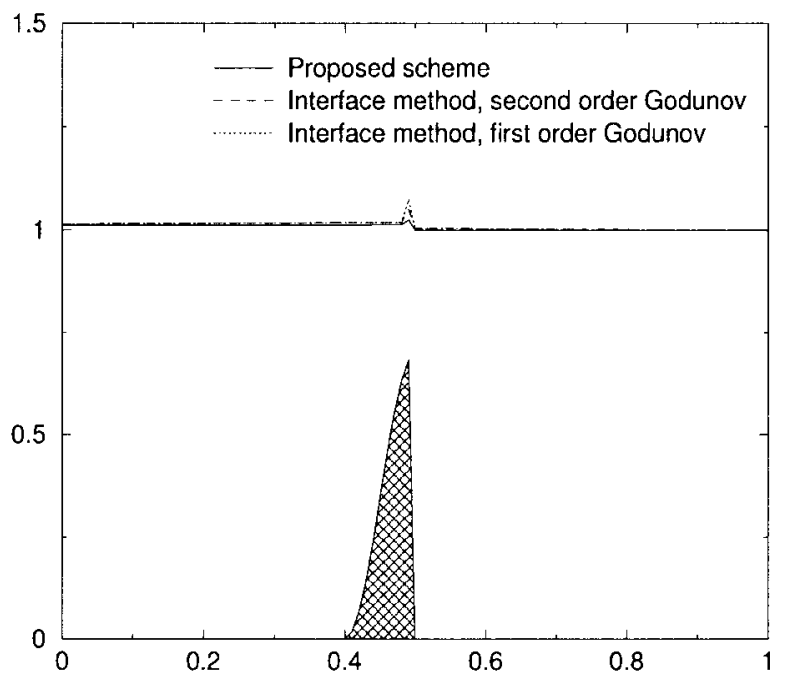

Figure 7 . Test $4.2: \frac{1}{2}\left(\frac{q}{h}\right)^{2}+g(h+z(x))$ for the proposed scheme and the first and second order Godunov interface method.

TABLE 1. Errors in $L^{1}$ and $L^{\infty}$ norms.

\begin{tabular}{|l|c|c|c|}
\hline & Proposed scheme & First order God. Interf. & Second order God. Interf. \\
\hline$L^{1}$ & $7 \times 10^{-3}$ & $7.8 \times 10^{-3}$ & $7.5 \times 10^{-3}$ \\
\hline$L^{\infty}$ & $1.9 \times 10^{-4}$ & $7 \times 10^{-4}$ & $4.5 \times 10^{-4}$ \\
\hline
\end{tabular}

We start from the numerical initial condition,

$$
\begin{gathered}
h_{i}=1-\frac{1}{2}\left(z_{i-1 / 2}+z_{i+1 / 2}\right) ; \\
q_{i}=0.1 h_{i} .
\end{gathered}
$$

For this test, we reach the exact solution given by

$$
\frac{1}{2}\left(\frac{q}{h}\right)^{2}+g(h+z(x))=1.005 .
$$

In Figure 7 we compare the numerical solutions provided by the proposed scheme with the first and second order Godunov inteface method (using slope limiters) proposed in [12]. In Figure 8 we compare also with the exact solution in an amplified region of the domain.

In Table 1, we present the errors corresponding to the first and second order Godunov interface methods and our scheme, in $L^{1}$ and $L^{\infty}$ norms. We observe that our scheme provides an improvement of even the second order Godunov method.

Test 5. Dry zone occurrence by a double rarefaction

In this test, we study the performance of the proposed scheme when there are some dry areas in the flow $(h=0)$. This test has been proposed in [8]. We use the nearly exact solution introduced in [3] to compare with the results of our proposed scheme.

The bottom function is

$$
z(x)=\left\{\begin{array}{cc}
1 & 25 / 3<x<12.5 \\
0 & \text { otherwise }
\end{array}\right.
$$




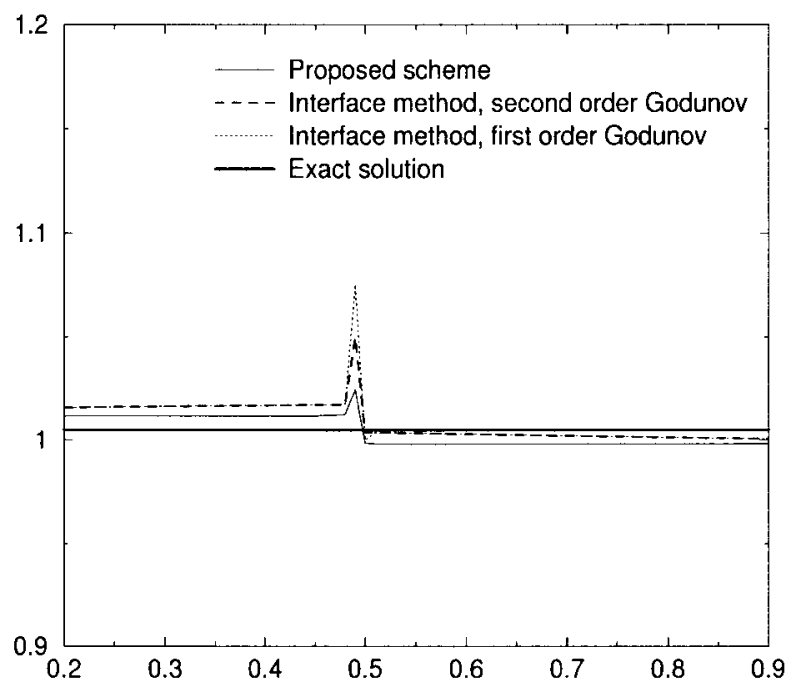

Figure 8. Test 4.2: Zoom of Figure 7.

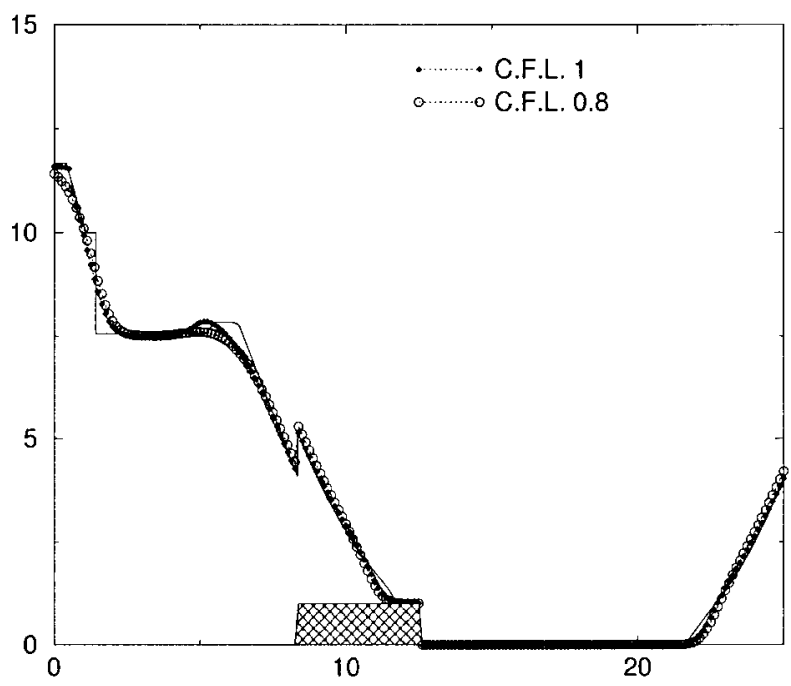

Figure 9. Test 5: Dry zone occurrence by a double rarefaction.

The boundary conditions are $\partial_{x} h=0, \partial_{x} q=0$, and as initial conditions we take

$$
\begin{gathered}
h_{i}=10-z\left(x_{i}\right) ; \\
q_{i}=\left\{\begin{array}{c}
350 \text { if } x_{i}>50 / 3 ; \\
-350 \quad \text { otherwise. }
\end{array}\right.
\end{gathered}
$$

In Figure 9 we present the numerical result for $t=0.25$, for C.F.L. numbers equal to 0.8 and 1 . We observe that the occurence of the dry zone is well solved $(h=0)$ and does not originate instabilities in our results.

Test 6. Steady solution with shock 


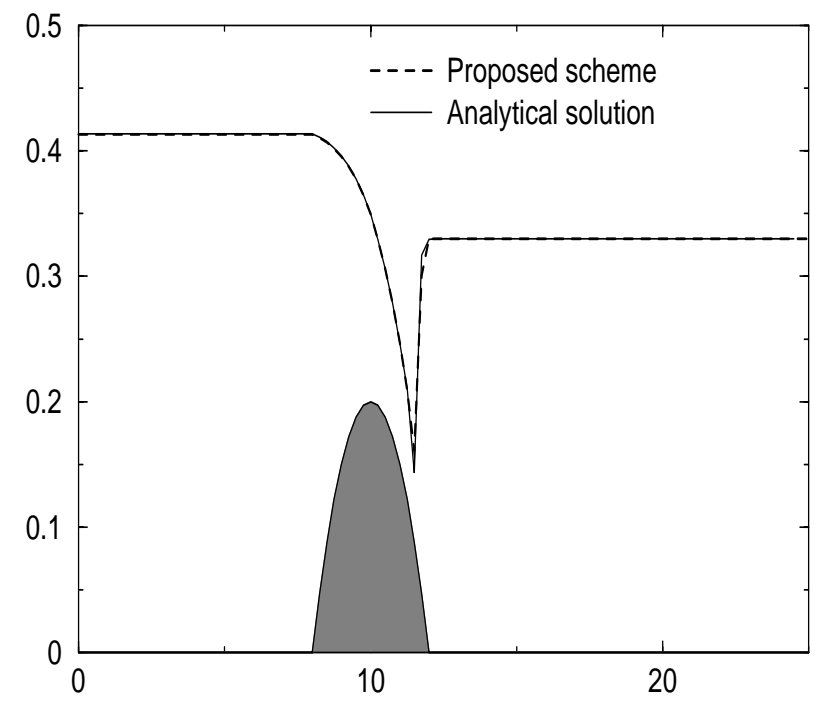

Figure 10. Test 6: Transcritical flow with shock: Free Surface.

In this test we calculate a steady flow over a bump defined by

$$
z(x)=\left\{\begin{array}{lc}
0.2-0.05(x-10)^{2} ; & \text { if } 8<x<12 \\
0 & \text { otherwise }
\end{array}\right.
$$

The channel length is $L=28 \mathrm{~m}$. The boundary conditions are

$$
\begin{cases}\frac{\partial h}{\partial x}=0, \quad q=1.53 & \text { at } x=0 \\ h=0.33, \quad \frac{\partial q}{\partial x}=0 & \text { at } x=28\end{cases}
$$

This test has been solved in [21] and [23] with several techniques.

We have taken $\Delta x=0.25 \mathrm{~m}$ and a CFL condition equal to 0.8. In Figure 10 we observe that our method is close to the analytical solution. In Figure 11 we compare our method with Roe's without Harten Regularization. We observe the "dog leg" profile produced by the presence of a sonic point when no Harten regularization is applied. This generates some inaccuracies that propagate upstream.

Test 7. Riemann problem with variable depth

This test consists in a Riemann problem with variable bottom, but with a larger jump of depth and discharge. The bottom function is

$$
z(x)= \begin{cases}0.25(\cos (\pi(x-0.5) / 0.1)+1) & \text { if } 0.4<x<0.6 \\ 0 & \text { otherwise }\end{cases}
$$

The channel length is $L=1 \mathrm{~m}$. We have taken $\Delta x=0.01 \mathrm{~m}$ and a CFL condition equal to 0.8. As initial condition we have considered

$$
W(x, 0)=\left\{\begin{array}{cll}
h=11, & q=20 & \text { for } x<0.4 \\
h=1, & q=0 & \text { for } x>0.4 .
\end{array}\right.
$$

The results are presented for a final time $t=0.02 \mathrm{~s}$. In Figure 12 we test our scheme versus Roe's scheme with Harten regularization with parameter $\epsilon=0.5$. It is observed as, near the shock (located near $x=0.63$ ) this 

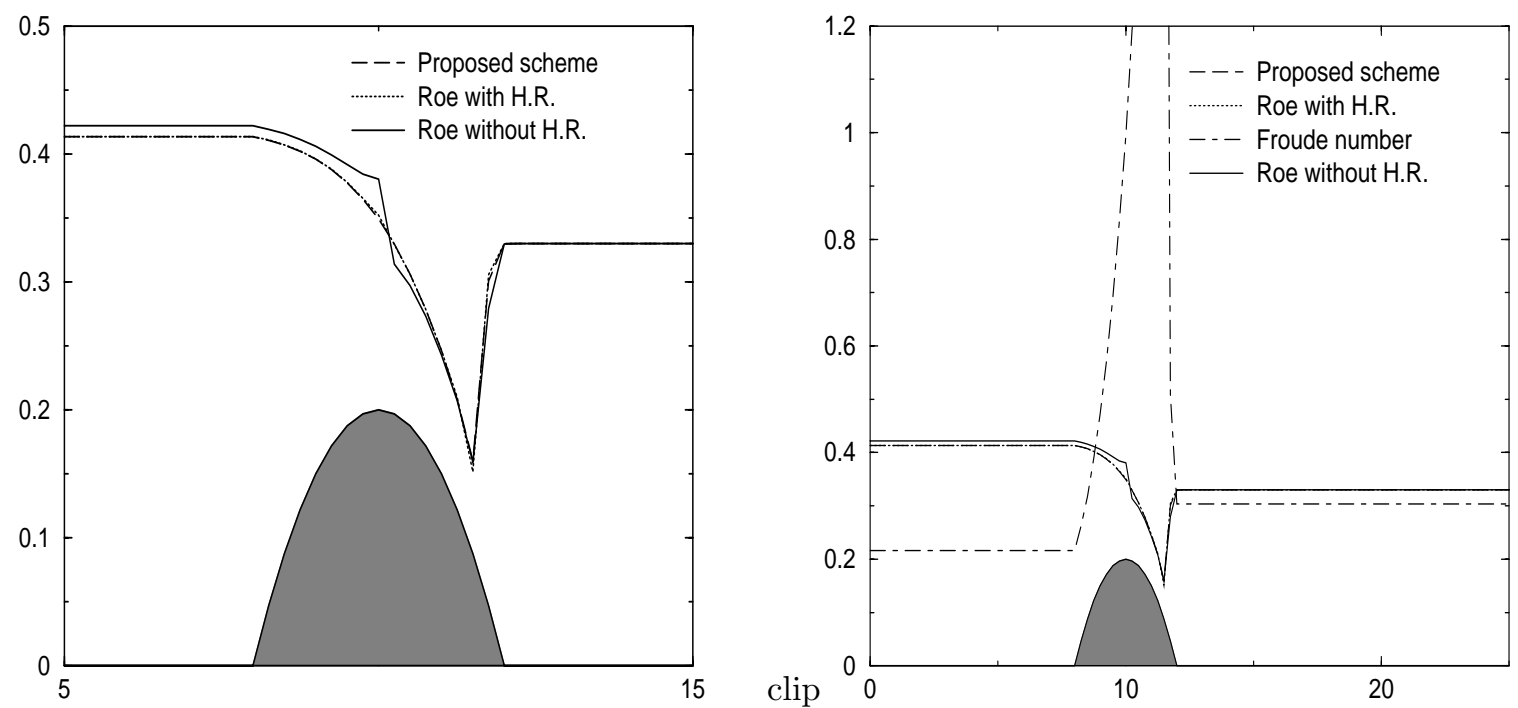

Figure 11. Test 6: Transcritical flow with shock: Free Surface.
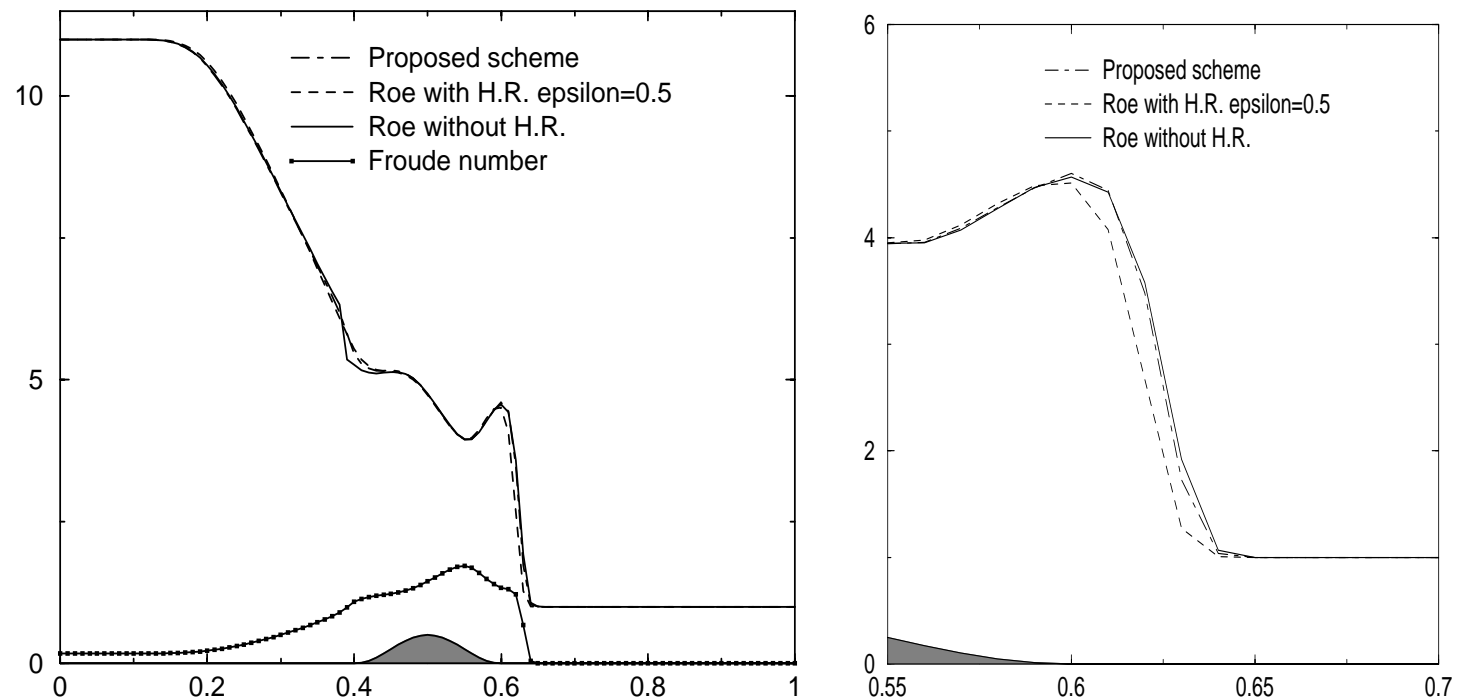

Figure 12. Free Surface for Test 7: Proposed scheme versus Harten regularization with $\epsilon=0.5$. Left: whole channel. Right: zoom near shock.

method introduces a larger diffusion, producing a loss of accuracy; while the proposed method gives a better fitting of the shock.

In Figure 13 we have used as regularization parameter $\epsilon=0.1$. In this case the diffusion on the shock diminishes, but both Roe's and Van Leer's methods lose precision near the sonic point, in a similar way to the case when Harten regularization is not used.

In Figure 14 is used the value $\epsilon=0.25$, that produces a result very close to our scheme. 

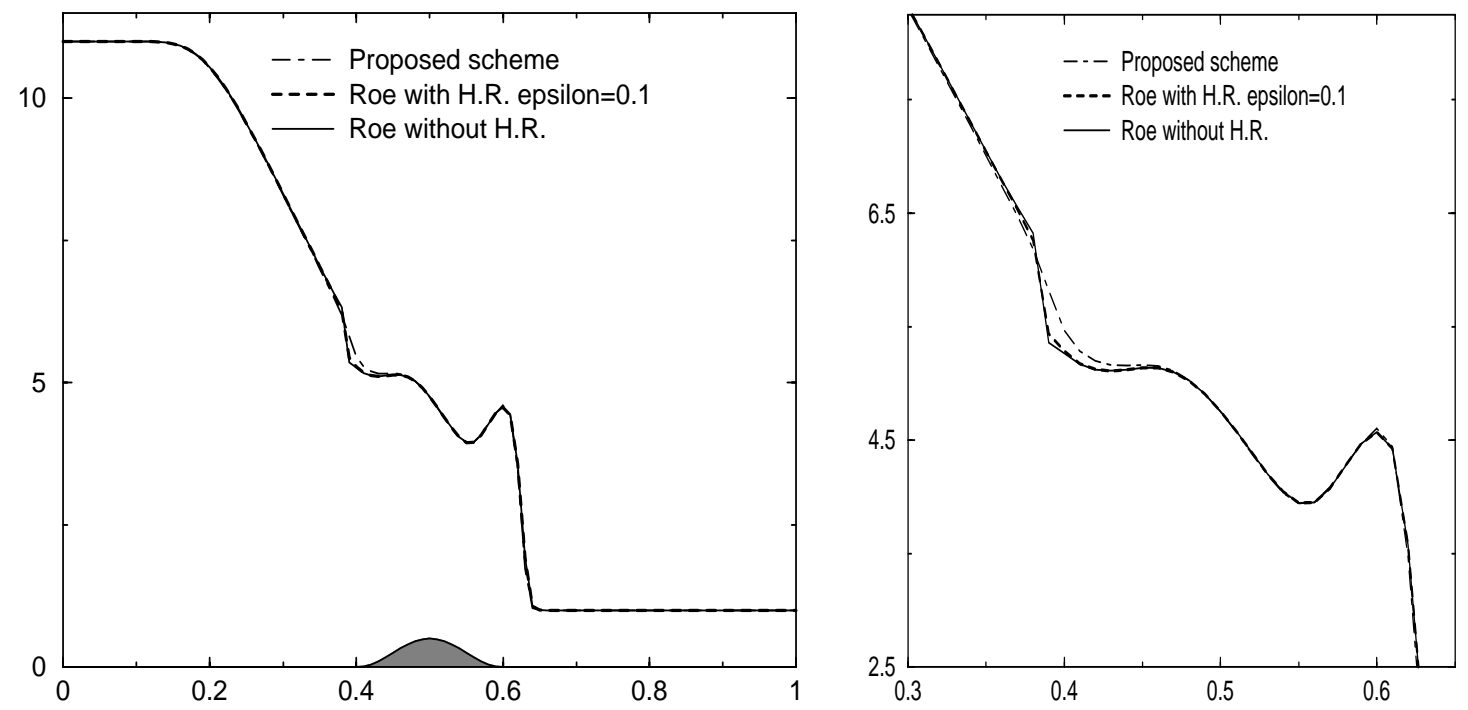

Figure 13. Free surface for Test 7: Proposed scheme versus Harten regularization with $\epsilon=0.1$. Left: whole channel. Right: zoom near sonic point.

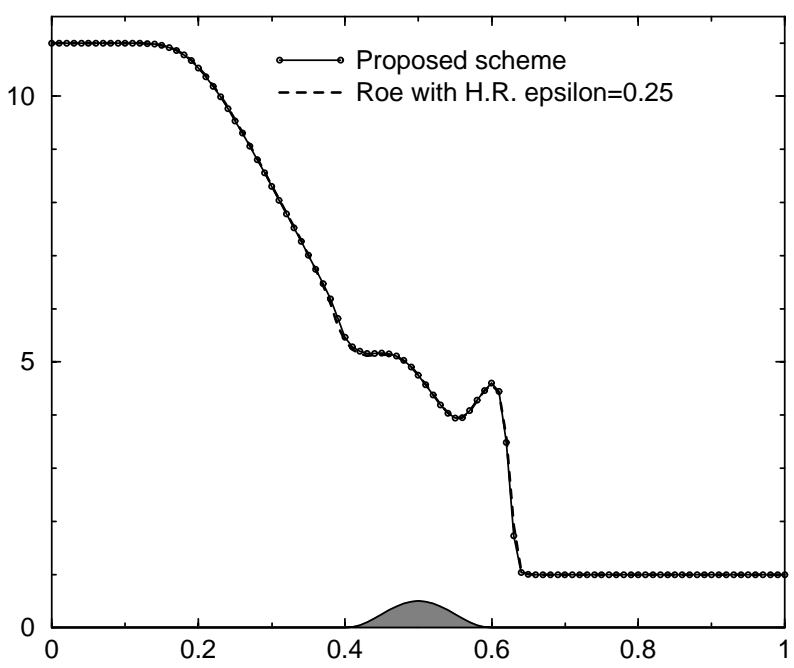

Figure 14. Free surface for Test 7: Proposed scheme versus Harten regularization with $\epsilon=0.25$.

\section{CONCLUSION}

In this paper we have introduced a hybrid flux-difference - flux-splitting solver for the 1D SWE with source term. The main methodological innovations are firstly, to use an upwinding matrix whose eigenvalues become zero in the supercritical zone only, and secondly to systematically introduce specific decentered discretizations of the source term that solve up to second order all equilibria solutions. The proposed method does not need any kind of regularization to avoid loosing accuracy near critical points while introducing low levels of numerical diffusion across shocks. 
Acknowledgements. The authors wish to thank Professors Manuel J. Castro Díaz, Macarena Gómez Mármol, Carlos Parés Madroñal, Benoit Perthame and María Elena Vázquez Cendón for their interest in the development of this work.

\section{REFERENCES}

[1] A. Bermudez, A. Dervieux, J.A. Desideri and M.E.V. Cendón, Upwind schemes for the two-dimensional shallow water equations with variable depth using unstructured meshes. Comput. Methods Appl. Mech. Engrg. 155 (1998) 49-72.

[2] A. Bermúdez and M.E.V. Cendón, Upwind Methods for Hyperbolic Conservation Laws with Source Terms. Comput. \& Fluids 23 (1994) 1049-1071.

[3] F. Bouchut, An introduction to finite volume methods for hyperbolic systems of conservation laws with source. Actas Ecole CEA-EDF-INRIA, Free surface geophysical flows, 7-10 Octobre, INRIA Rocquencourt, France (2002).

[4] F. Dubois and G. Mehlman, A non-parameterized entropy correction for Roe's approximate Riemann solver. Numer. Math. 73 (1996) 169-208.

[5] P. Brufau, Simulación bidimensional de flujos hidrodinámicos transitorios en gemotrías irregulares. Ph.D. thesis, Universidad de Zaragoza (2000).

[6] T.C. Rebollo, E.D.F. Nieto and M.G. Mármol, A flux-splitting solver for shallow watter equations with source terms. Int. J. Num. Methods Fluids 42 (2003) 23-55.

[7] T.C. Rebollo, A.D. Delgado and E.D.F. Nieto, A family of stable numerical solvers for Shallow Water equations with source terms. Comput. Methods Appl. Mech. Engrg. 192 (2003) 203-225.

[8] T. Gallouët, J.-M. Hérard and N. Seguin, Some approximate Godunov schemes to compute shallow-water equations with topography. Comput. \& Fluids 32 (2003) 479-513.

[9] E. Godlewski and P.A. Raviart, Hyperbolic systems of conservation laws. Math. Appl. (1991).

[10] E. Godlewski and P.A. Raviart, Numerical Approximation of Hyperbolic Systems of Conservation Laws. Springer-Verlag (1996).

[11] A. Harten, P. Lax and A. Van Leer, On upstream differencing and Godunov-type scheme for hyperbolic conservation laws. SIAM Rev. 25 (1983) 35.

[12] S. Jin, A steady-state capturing method for hyperbolic systems with geometrical source terms. ESAIM: M2AN 35 (2001) 631-645.

[13] A. Kurganov and D. Levy, Central-upwind schemes for the saint-venant system. ESAIM: M2AN 36 (2002) 397-425.

[14] A. Kurganov and E. Tadmor, New High-Resolution Central Schemes for Nonlinear Conservations Laws and ConvectionDiffusion Equations. J. Comput. Phys. 160 (2000) 214-282.

[15] R.J. Le Veque and H.C. Yee, A study of numerical methods for hyperbolic conservation laws with stiff source terms. J. Comput. Phys. 86 (1990) 187-210.

[16] R.J. Le Veque, Balancing Source Terms and Flux Gradients in High-Resolution Godunov Methods: The Quasi-Steady Wave-Propagation Algorithm. J. Comp. Phys. 146 (1998) 346-365.

[17] B. Perthame and C. Simeoni, A kinetic scheme for the Saint-Venant system with a source term. Calcolo 38 (2001) $201-231$.

[18] P.L. Roe, Upwind differencing schemes for hyperbolic conservation laws with source terms, in Nonlinear Hyperbolic Problems, C. Carraso, P.A. Raviart and D. Serre, Eds., Springer-Verlag, Lecture Notes in Math. 1270 (1986) 41-51.

[19] E.F. Toro, Riemann Solvers and Numerical Methods for Fluid Dynamics. Springer (1997).

[20] M.E.V. Cendon, Estudio de esquemas descentrados para su aplicacion a las leyes de conservación hiperbólicas con términos fuente. Ph.D. thesis, Universidad de Santiago de Compostela (1994).

[21] M.E.V. Cendón, Improved Treatment of Source Terms in Upwind Schemes for the Shallow Water Equations in Channels with Irregular Geometry. J. Comp. Phys. 148 (1999) 497-526.

[22] J.P. Vila, High-order schemes and entropy condition for nonlinear hyperbolic systems of conservations laws. Math. Comp. 50 (1988) 53-73.

[23] J.G. Zhou, D.M. Causon, C.G. Mingham and D.M. Ingram, The Surface Gradient Method for the Treatment of Source Terms in the Sallow-Water Equations. J. Comput. Phys. 168 (2001) 1-25. 